\title{
A study on the effects of organizational structure on success of performance measurement
}

\author{
Davood Khaleghi ${ }^{\mathrm{a}^{*}}$, Hossein Akhavan Alavi ${ }^{\mathrm{b}}$ and Mostafa Alimiric
}

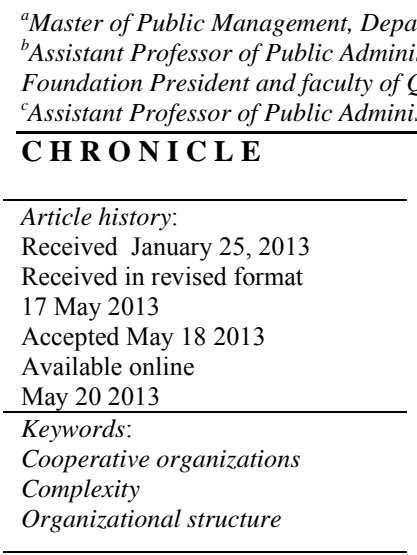

\begin{abstract}
Organizational structure is one of the most important components of any firm and it plays essential role on building successful business units. Organizational structure in cooperative organizations may influence productivity of such organizations, significantly. In this paper, we consider the relationship between various components including formality, complexity, concentration on performance measurement in cooperative organizations in province of Qom, Iran. The study selects 280 firms out of 1069 cooperative organizations and using Pearson correlation test examines different hypotheses. Based on the results of our survey, formality and concentration have positive impacts on the success of cooperative organizations but the impact of complexity on performance of organization is not confirmed.
\end{abstract}

(C) 2013 Growing Science Ltd. All rights reserved.

\section{Introduction}

Organizational structure plays important role on the success of organizations. A successful organization normally uses a horizontal and less complicated structure. There are literally different studies associated with the effects of organizational structure on the success of organizations. Tahernezhad et al. (2013) investigated the relationship between organizational structure and learning characteristics in a case study of revenue agency located in province of Alborz, Iran. In this study, organizational structure incorporated five items of complexity, concentration, authority, formality and flexibility. They reported that there were some meaningful relationships between three variables of formality, complexity and authority on one side and learning capability from the other side. However, the study did not find any meaningful relationship between concentration and flexibility with organizational learning capability. 
Khalghani et al. (2013) studied organizational structure, culture, and information technology as knowledge management (KM) infrastructural capabilities, and compares their effects in five medical research centers in Tehran, Iran. They reported that in terms of their status quo, the three studied KM enablers were at various conditions, with organizational culture having the best (mean rank=1.79) and IT the worst (mean rank=2.14) status. Moreover, it was disclosed by regression analysis that organizational structure had the most significant impact (Beta $=0.397$ ) on the effectiveness of knowledge management initiatives, while information technology gained the least perceived impact $($ Beta $=0.176)$.

Fathizadeh et al. (2012) studied the relationship between organizational structure and organizational agility in an insurance company in Iran. They concluded that there was a significant relationship between organizational agility and two dimensions of organizational structure, i.e. formalization and centralization and they reported no significant relationship between complexity and organizational agility. Ali et al. (2012) examined organizational design features implemented by firms in pursuing their sustainable supply chain objectives. The research purpose was to gain a better understanding of the organizational design features, which firms currently implement or may apply in the future. The results should encourage organizations to address design issues as they would be associated with overall supply chain effectiveness. The ever-increasing impacts of the wider perspectives such as the pursuit of sustainability drive for industry consolidation/rationalization and the requirements for responding to changing customer preferences could mean the conventional wisdom of organizing for success was increasingly becoming grossly inadequate, if not useless. There were numerous reasons why companies start to rethink about organizational design, organizational structure and its performance to attain a supply chain sustainability journey.

Darvish et al. (2013a) investigated the impacts of intellectual capital on organizational performance measurement through organizational learning capabilities. Wei Phang et al. (2008) investigated organizational learning in eGovernment projects using a multi-theoretic approach. Hanzaee and Mirvaisi (2013) performed a survey on effects of emotional intelligence, organizational citizenship behaviors and job satisfaction on employees' performance in Iranian hotel industry. Darvish et al. (2013b) investigated the effect of two internal and external factors on dynamic organizational skills through information technology equipment. The study selected a sample of 52 experts and using a questionnaire, they gathered some insight about the proposed study based on the implementation of structural equation modeling. The results confirmed that both internal and external factors influenced dynamic organizational skills through information technology equipment. The study also used freedman test to rank the factors and the results demonstrated that communication was the most important factor (4.41), followed by process (4.03), knowledge implementation (2.79), decision making (2.54) and human resources (1.22) was the last important factor.

\section{The proposed study}

In this paper, we consider the relationship between various components including formality, complexity, concentration on performance measurement in cooperative organizations in province of Qom, Iran. The study uses the following formula to calculate the minimum number of sample size,

$n=\frac{N \times z_{\alpha / 2}^{2} \times p \times q}{\varepsilon^{2} \times(N-1)+z_{\alpha / 2}^{2} \times p \times q}$,

where $N$ is the population size, $p=1-q$ represents the yes/no categories, $z_{\alpha / 2}$ is CDF of normal distribution and finally $\varepsilon$ is the error term. Since we have $p=0.5, z_{\alpha / 2}=1.96$ and $N=1069$, the number of sample size is calculated as $n=280$. There are three hypotheses associated with the proposed study of this paper considers the following three hypotheses as follows, 
1. There is a meaningful relationship between formality and performance measurement of cooperative firms.

2. There is a meaningful relationship between concentration and performance measurement of cooperative firms.

3. There is a meaningful relationship between complexity and performance measurement of cooperative firms.

Fig. 1 demonstrates the structure of the proposed study of this paper.

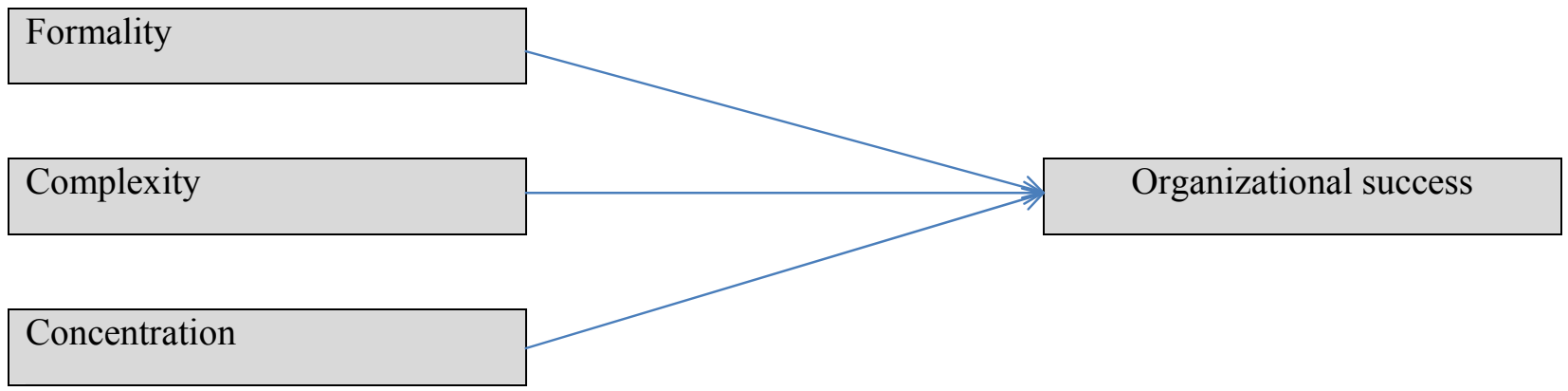

Fig. 1. The proposed study

\section{The results}

In this section, we present details of our findings on testing three hypotheses of this survey based on Pearson correlation as well as regression analysis. Table 1 demonstrates the results of our findings on the implementation of Pearson correlation test.

\section{Table 1}

The results of Pearson correlation test

\begin{tabular}{lccl}
\hline Component & Pearson ratio & P-value & Results \\
\hline Formality-organizational success & 0.71 & 0.001 & Confirmed \\
Complexity-organizational success & -0.65 & 0.1 & Reject \\
Concentration-organizational success & 0.83 & 0.001 & Confirmed \\
\hline
\end{tabular}

As we can observe from the results of Table 1, there are some meaningful relationship between two independent variables, formality and concentration and organization success. We have also performed a regression analysis between three variables and dependent variable, performance measurement.

Table 2

The summary of regression analysis

\begin{tabular}{llllll}
\hline & \multicolumn{4}{l}{ Unstandardized Coefficients } & \multicolumn{2}{l}{ Standardized Coefficients } & \\
Variable & Coefficient & Standard error & Coefficient & t-value & P-value \\
\hline Intercept & 0.563 & 0.401 & & 3.897 & 0.01 \\
Formality & 0.209 & 0.167 & 0.198 & 7.031 & 0.00 \\
Complexity & -0.117 & 0.271 & -0.167 & 3.771 & 0.1 \\
Concentration & 0.239 & 0.103 & 0.253 & 5.787 & 0.001 \\
\hline
\end{tabular}


The results of Table 2 demonstrate that formality and concentration are statistically significance but complexity when $\alpha=0.05$.

\section{Discussion and conclusion}

There is no doubt that an efficient and productive business unit is capable of contributing more of economy and there is a better social welfare on working for such organizations. Cooperative organizations have been considered as a bridge to build a good alliance among small investors who wish to build a working business in todays' economy. However, cooperative organizations must incorporate suitable structure to help members have good cooperation. In this paper, we have presented an empirical survey to measure the impact of formality, complexity and concentration on performance of cooperative organizations. The results of our investigation have concluded that while there were some strong and positive effects from formality and concentration on organizational performance there was no indication of any possible effect from complexity on organizational performance.

\section{Acknowledgment}

The authors would like to thank the anonymous referees for constructive comments on earlier version of this work, which have contributed to quality of the work.

\section{References}

Ali, S., Alam, M \& Bhowmick, S. (2012). Organizational design in the context of supply chain sustainability: A conceptual model. Management Science Letters , 2(4), 1141-1154.

Darvish, H., Ahmadi, A.A., Kafashzadeh, A.R., \& Farid, S., \& Nejatizadeh, N.O. (2013a). Investigating the effects of intellectual capital on organizational performance measurement through organizational learning capabilities. Management Science Letters, 3(1), 165-172.

Darvish, H., Dehkordi, L., Saremi, M., Kafashzadeh, A \& Faradonbeh, M. (2013b). An empirical study to investigate the effects of internal and external knowledge management on dynamic organizational skills through information technology tools. Management Science Letters, 3(4), 1239-1244.

Fathizadeh, A., Ahmadi, S., Sadeghi, J., daryabeig, M \& Taherkhani, L. (2012). A study on the relationship between organizational structure and organizational agility: A case study of insurance firm. Management Science Letters, 2(8), 2777-2788.

Hanzaee, K \& Mirvaisi, M. (2013). A survey on impact of emotional intelligence, organizational citizenship behaviors and job satisfaction on employees' performance in Iranian hotel industry. Management Science Letters, 3(5), 1395-1402.

Khalghani, A., Reshadatjoo, H \& Iran-nejad-parizi, M. (2013). A study on organizational culture, structure and information technology as three KM enablers: A case study in five Iranian medical and healthcare research centers. Management Science Letters, 3(1), 147-158.

Tahernezhad, K., Sadaghiani, J \& Ghaiyoomi, A. (2013). A study on relationship between organizational structure and learning characteristics: A case study of revenue agency. Management Science Letters, 3(3), 991-994.

Wei Phang, C., Kankanhalli, A., \& Ang, C. (2008). Investigating organizational learning in eGovernment projects: A multi-theoretic approach. The Journal of Strategic Information Systems, $17(2), 99-123$. 